CARDIOVASCULAR MEDICINE

\title{
$N$-acetylcysteine does not prevent contrast induced nephropathy after cardiac catheterisation with an ionic low osmolality contrast medium: a multicentre clinical trial
}

\author{
V O Gomes, C E Poli de Figueredo, P Caramori, R Lasevitch, L C Bodanese, A Araújo, A P Röedel, \\ A P Caramori, F S Brito Jr, H G Bezerra, P Nery, A Brizolara
}

Heart 2005;91:774-778. doi: 10.1136/hrt.2004.039636

\begin{abstract}
See end of article for authors' affiliations

Correspondence to: Dr Paulo Caramori, Centre of Cardiovascular Research, Hospital São Lucas-PUCRS, Avenida lpiranga 6690 room 300 90610-000, Porto Alegre, RS, Brazil; caramori.p@ plug-in.com.br
\end{abstract}

Accepted 9 August 2004
Objective: To evaluate oral $\mathrm{N}$-acetylcysteine in the prevention of contrast induced nephropathy (CIN) in patients at low to moderate risk undergoing cardiac catheterisation with ionic low osmolality contrast medium.

Methods: In a multicentre double blind clinical trial 156 patients undergoing coronary angiography or percutaneous coronary intervention with serum creatinine $\geqslant 106.08 \mu \mathrm{mol} / \mathrm{l}$ or creatinine clearance $<50 \mathrm{ml} / \mathrm{min}$ or diabetes mellitus were randomly assigned to receive $\mathrm{N}$-acetylcysteine $600 \mathrm{mg}$ orally twice daily for two days or placebo. Only low osmolality ionic contrast medium was used.

Results: Sixteen patients developed CIN, defined as an increase of $44.2 \mu \mathrm{mol} / \mathrm{l}$ in creatinine in 48 hours: eight of 77 patients $(10.4 \%)$ in the $N$-acetylcysteine group and eight of 79 patients $(10.1 \%)$ in the placebo group $(p=1.00)$. The mean (SD) change in serum creatinine was similar in both groups: 7.96 (35.36) $\mu \mathrm{mol} / \mathrm{l}$ in the $N$-acetylcysteine group and 6.19 (25.64) $\mu \mathrm{mol} / \mathrm{l}$ in the placebo group $(\mathrm{p}=0.67)$. No difference was observed in the change in endogenous creatinine clearance $(-0.54(10.4) \mathrm{ml} / \mathrm{min} v$ -2.52 (12.3) $\mathrm{ml} / \mathrm{min}, \mathrm{N}$-acetylcysteine and placebo, respectively, $\mathrm{p}=0.28$ ).

Conclusion: Oral $\mathrm{N}$-acetylcysteine did not prevent $\mathrm{CIN}$ in patients at low to moderate risk undergoing cardiac catheterisation with ionic low osmolality contrast medium.
O ne of the most frequent complications of interventional cardiac procedures is contrast induced nephropathy (CIN).

The development of CIN has been associated with increases in the length of hospitalisation, ${ }^{1}$ morbidity, and mortality. ${ }^{2}$ Although several preventive measures have been studied, only a few, such as hydration ${ }^{4}$ and use of low osmolality contrast media, ${ }^{5}$ have been shown to be useful. There is evidence that the two principal independent risk factors for CIN are impaired renal function and diabetes mellitus. ${ }^{2}$

Although the mechanisms of contrast induced renal injury are not fully understood, there is evidence that contrast media cause renal vasoconstriction ${ }^{67}$ with consequent hypoxia and renal injury. In addition, free radical release, associated with exposure to contrast media, may play an important part by causing direct damage to the renal tubular epithelium. $^{8}{ }^{9}$

$\mathrm{N}$-acetylcysteine has the potential to prevent CIN due to its potent antioxidant and vasodilating actions secondary to increased nitric oxide synthase expression. ${ }^{7} \mathrm{~N}$-acetylcysteine was shown to be effective in preventing CIN in non-cardiac patients with previously impaired renal function undergoing contrast enhanced computed tomography with a small volume of intravenous contrast. ${ }^{10}$ However, in clinical trials with patients undergoing cardiac catheterisation the effects of oral $N$-acetylcysteine have not been consistently beneficial. ${ }^{11-19}$ Recently, a meta-analysis of studies of non-ionic low osmolality contrast media suggested that $\mathrm{N}$-acetylcysteine can be useful in preventing CIN in patients undergoing cardiac catheterisation. ${ }^{20}$ To our knowledge, no published clinical trial has investigated the role of $N$-acetylcysteine in preventing CIN in patients undergoing radiographic procedures with an ionic low osmolality contrast media.

This was a multicentre, randomised, double blind, placebo controlled clinical trial, carried out to evaluate the effect of oral $\mathrm{N}$-acetylcysteine on preventing CIN in patients at low to moderate risk undergoing cardiac catheterisation with the use of an ionic low osmolality contrast medium.

\section{METHODS}

\section{Patients}

Patients at risk for developing CIN who were referred for elective coronary angiography or percutaneous coronary intervention (PCI) at four Brazilian hospitals were enrolled in the present study.

Patients were considered to be at risk for developing CIN if they had one of the following criteria: serum creatinine $\geqslant 106.08 \mu \mathrm{mol} / \mathrm{l}$, creatinine clearance $(\mathrm{CrCl})<50 \mathrm{ml} / \mathrm{min}$, or drug treated diabetes mellitus. Exclusion criteria were age under 18 years, use of radiographic contrast media within 21 days of randomisation, current dialysis, haemodynamic instability before the procedure (systolic blood pressure $\leqslant 90 \mathrm{~mm} \mathrm{Hg}$ or diastolic blood pressure $\leqslant 60 \mathrm{~mm} \mathrm{Hg}$ ), and history of sensitivity to $N$-acetylcysteine.

$\mathrm{CrCl}$ was estimated with the Cockcroft-Gault formula, where $\mathrm{CrCl}=([140-$ age $] \times$ weight $(\mathrm{kg}) /$ serum creatinine $(\mathrm{mg} / \mathrm{dl}) \times 72)$, with adjustment for female sex $\left(\mathrm{CrCl}_{\text {female }}=\mathrm{CrCl} \times 0.85\right) .{ }^{21}$ The ethics committees of the participant centres approved the study protocol. Written informed consent was obtained from all patients.

\section{Study protocol}

Patients were randomly assigned on a 1:1 basis to receive $N$ acetylcysteine or placebo by randomly drawing sealed envelopes containing either the active drug or matching placebo. The randomisation sequence was computer

Abbreviations: $\mathrm{Cl}$, confidence interval; $\mathrm{CIN}$, contrast induced nephropathy; $\mathrm{CrCl}$, creatinine clearance; $\mathrm{PCl}$, percutaneous coronary intervention 
generated and was stratified by the procedure to be performed (coronary angiography or PCI). $\mathrm{N}$-acetylcysteine and placebo envelopes were prepared by the industrial pharmacy at the headquarters (Hospital São Lucas-PUCRS). The investigators were not aware of the contents of the envelopes until the end of the study. $N$-acetylcysteine was orally administered at the dose of $600 \mathrm{mg}$ twice a day, starting one day before the procedure (two doses before and two doses after the procedure). All patients received intravenous saline $0.9 \% 1 \mathrm{ml} / \mathrm{kg} / \mathrm{h}$ from 12 hours before to 12 hours after exposure to the contrast medium. Serum creatinine was measured 48 hours before (baseline creatinine) and 48 hours after the procedure (post-procedure creatinine). All the procedures were performed with the low osmolality ionic contrast medium ioxaglate (Hexabrix; Guerbet Ltda, Rio de Janeiro, Brazil).

\section{End points}

The primary end points of the study were: (1) the occurrence of CIN, defined as an increase in serum creatinine $\geqslant 44.2 \mu \mathrm{mol} / \mathrm{l}, 48$ hours after exposure to contrast medium; (2) the change in serum creatinine (post-procedure creatinine - baseline creatinine); and (3) the change in $\mathrm{CrCl}$ (postprocedure $\mathrm{CrCl}-$ baseline $\mathrm{CrCl}$ ). Secondary end points were in-hospital death, need for haemodialysis, and length of hospitalisation.

The effect of $\mathrm{N}$-acetylcysteine on the following predefined subgroups was also evaluated: diabetes mellitus, baseline creatinine $\geqslant 132.6 \mu \mathrm{mol} / \mathrm{l}$, and volume of the contrast medium used in the procedure $\leqslant 1.5 \mathrm{ml} / \mathrm{kg}$.

\section{Statistical analysis}

Categorical variables were compared by the $\chi^{2}$ test or Fischer's test. Continuous variables were compared by two tailed unpaired Student's $t$ test. Data are expressed as mean (SD). For variables with non-parametric distribution, the Mann-Whitney test was used and data are expressed as median (25th to 75 th centile). Multivariate analysis was performed to adjust for the difference in baseline creatinine between groups by assessing the primary outcomes.

On the basis of an expected $10 \%$ incidence of CIN in this study population, ${ }^{12}{ }^{14}$ a two tailed significance level of 0.05 ,
Table 2 Incidence of contrast medium induced nephropathy and changes in serum creatinine and clearance of endogenous creatinine from baseline to 48 hours after contrast medium exposure

\begin{tabular}{llll}
\hline & $\begin{array}{l}\text { N-acetylcysteine } \\
(\mathbf{n}=77)\end{array}$ & $\begin{array}{l}\text { Placebo } \\
(\mathbf{n}=79)\end{array}$ & p Value \\
\hline Incidence of $\mathrm{CIN}$ & $8(10.4 \%)$ & $8(10.1 \%)$ & 1.00 \\
Change in creatinine $(\mu \mathrm{mol} / \mathrm{l}) 7.96(35.36)$ & $6.19(25.64)$ & 0.67 \\
Change in $\mathrm{CrCl}(\mathrm{ml} / \mathrm{min})$ & $-0.43(10.6)$ & $-2.82(12.0)$ & 0.19 \\
\hline
\end{tabular}

Values are mean (SD).

$\mathrm{CIN}$, contrast induced nephropathy.

and a statistical power of $80 \%$, a sample size of 156 patients would be required to detect a $50 \%$ change in the relative risk for CIN associated with the active treatment. Data were analysed with SPSS software, version 10.1 (SPSS Inc, Chicago, Illinois, USA).

\section{RESULTS}

From April 2001 to April 2003, 156 patients were enrolled in the study to receive $N$-acetylcysteine (77 patients) or placebo (79 patients). Table 1 shows the baseline clinical characteristics and procedure related variables. The mean (SD) baseline serum creatinine and $\mathrm{CrCl}$ of the study population were 117.57 (39.78) $\mu \mathrm{mol} / \mathrm{l}$ and 60.9 (29.5) $\mathrm{ml} / \mathrm{min}$, respectively.

Groups were well balanced with respect to age, sex, body mass index, blood pressure, diabetes mellitus, and drug treatment. In the placebo group, serum creatinine was

Table 3 Secondary outcomes

\begin{tabular}{llll}
\hline & $\begin{array}{l}\text { N-acetylcysteine } \\
\text { (n=77) }\end{array}$ & $\begin{array}{l}\text { Placebo } \\
\text { (n=79) }\end{array}$ & p Value \\
\hline Length of hospitalisation (days) & $6(2-13)$ & $5(2-10)$ & 0.44 \\
Haemodialysis (n) & 2 & 0 & 0.24 \\
Death (n) & 5 & 2 & 0.42 \\
\hline
\end{tabular}

The numbers in parentheses are the 25 th and 75 th centiles.

\begin{tabular}{|c|c|c|c|}
\hline & N-acetylcysteine $(n=77)$ & Placebo $(n=79)$ & $\mathrm{p}$ Value \\
\hline Age (years) & $63.8(11.30)$ & $66.5(11.1)$ & 0.14 \\
\hline Male sex (\%) & 61.0 & 57.0 & 0.72 \\
\hline Body mass index $\left(\mathrm{kg} / \mathrm{m}^{2}\right)$ & $27.0(4.74)$ & $28.1(4.93)$ & 0.14 \\
\hline Creatinine $(\mu \mathrm{mol} / \mathrm{l})$ & $123.76(45.08)$ & 111.38 (30.94) & 0.05 \\
\hline $\mathrm{CrCl}(\mathrm{ml} / \mathrm{min})$ & $59.0(27.4)$ & $62.9(31.1)$ & 0.41 \\
\hline \multicolumn{4}{|l|}{ Blood pressure $(\mathrm{mm} \mathrm{Hg})$} \\
\hline Systolic & $131.8(19.4)$ & $131.3(23.2)$ & 0.90 \\
\hline Diastolic & $76.7(12.4)$ & $77.6(11.3)$ & 0.63 \\
\hline Hypertension & $87.0 \%$ & $84.8 \%$ & 0.86 \\
\hline Diabetes mellitus & $51.9 \%$ & $51.9 \%$ & 1.00 \\
\hline \multicolumn{4}{|l|}{ Drugs in use } \\
\hline Diuretics & $41.6 \%$ & $51.9 \%$ & 0.25 \\
\hline Calcium channel blocker & $10.4 \%$ & $15.2 \%$ & 0.51 \\
\hline ACE inhibitor & $70.1 \%$ & $68.4 \%$ & 0.94 \\
\hline Angiographic findings & & & 0.52 \\
\hline Non-obstructive disease & $12.9 \%$ & $14.0 \%$ & \\
\hline Single vessel disease & $21.4 \%$ & $31.0 \%$ & \\
\hline Double vessel disease & $28.6 \%$ & $26.8 \%$ & \\
\hline Triple vessel disease & $37.1 \%$ & $28.2 \%$ & \\
\hline LVEF (\%) & 55.3 & 59.4 & 0.30 \\
\hline Cardiac angiographic procedure & & & 1.00 \\
\hline Coronary angiography & $76.7 \%$ & $77.3 \%$ & \\
\hline Percutaneous intervention & $23.3 \%$ & $22.7 \%$ & \\
\hline Contrast volume (ml) & $102.5(47.3)$ & $102.8(60.4)$ & 0.97 \\
\hline
\end{tabular}

Values are mean (SD) or percentage.

$\mathrm{ACE}$, angiotensin converting enzyme; $\mathrm{CrCl}$, estimated creatinine clearance; $\mathrm{LVEF}$, left ventricular ejection fraction. 
slightly lower, with a borderline significance (111.38 (30.94) $v 123.76(45.08) \mu \mathrm{mol} / \mathrm{l}, \mathrm{p}=0.05)$. However, the estimated $\mathrm{CrCl}$ was similar in both groups (62.9 (31.1) v $59.0(27.4) \mathrm{ml} /$ $\min , \mathrm{p}=0.41$, respectively, for placebo and $N$-acetylcysteine). The volume of contrast used during the procedure and the proportion of coronary angiography and PCIs were similar in the groups. No adverse reaction to the study drug was reported.

\section{Primary end points}

The incidence of CIN was similar in both groups, as well as the change in serum creatinine and $\mathrm{CrCl}$ (table 2). After adjustment for baseline creatinine by logistic regression and multiple linear regression, as appropriate, the primary outcomes remained unchanged.

\section{Secondary end points}

No difference was observed between the groups regarding the secondary end points (table 3 ). The length of hospitalisation, need for haemodialysis, and incidence of in-hospital death were similar between groups. During the same hospitalisation 14 patients in the $\mathrm{N}$-acetylcysteine group and 10 in the placebo group underwent coronary artery bypass graft surgery after the cardiac catheterisation. Seven (4.6\%) patients died, five in the $N$-acetylcysteine group and two in the placebo group. In the $N$-acetylcysteine group, two (1.2\%) deaths were associated with the development of CIN and occurred in patients requiring haemodialysis. The three other deaths in the $\mathrm{N}$-acetylcysteine group were caused by stroke (one patient) and by postoperative complications of coronary artery bypass graft surgery (two patients). In the placebo group, one death was related to pulmonary thromboembolism and the other was associated with postoperative complications of coronary artery bypass graft surgery.

\section{Analysis of subgroups}

$N$-acetylcysteine provided no significant effect in any of the subgroups analysed (table 4). Among the 46 patients with serum creatinine $\geqslant 132.6 \mu \mathrm{mol} / \mathrm{l}$, the incidence of CIN was $15.4 \%$ in the $N$-acetylcysteine group and $20.0 \%$ in the placebo group (odds ratio (OR) $0.72,95 \%$ confidence interval (CI) 0.15 to $3.35 ; \mathrm{p}=0.71$ ).

Among the 81 patients with diabetes mellitus, the incidence of CIN was $10.0 \%$ in the $N$-acetylcysteine group and $9.8 \%$ in the placebo group (OR $1.02,95 \%$ CI 0.27 to 4.42 ; $\mathrm{p}=1.00)$.

Among the 96 patients receiving a low volume of contrast $(\leqslant 1.5 \mathrm{ml} / \mathrm{kg})$, the incidence of CIN in the $N$-acetylcysteine and placebo groups was $11.4 \%$ and $9.6 \%$, respectively (OR $1.20,95 \%$ CI 0.32 to $4.46 ; \mathrm{p}=1.00)$.

\section{DISCUSSION}

This is the first double blind, multicentre, randomised study assessing the effect of $\mathrm{N}$-acetylcysteine in preventing CIN in a population at low to moderate risk undergoing cardiac catheterisation with an ionic low osmolality contrast medium. Our findings indicate that oral $N$-acetylcysteine does not provide any benefit as compared with placebo to reduce the incidence of CIN in this patient population. In addition, the use of $\mathrm{N}$-acetylcysteine was not beneficial in any of the predefined subgroups of patients with diabetes, creatinine $>132.6 \mu \mathrm{mol} / \mathrm{l}$, and contrast volume $<1.5 \mathrm{ml} / \mathrm{kg}$. The lack of a beneficial effect of $N$-acetylcysteine was confirmed by multivariate analysis with control for the difference in the creatinine concentration before the procedure.

The initial study by Tepel and colleagues, ${ }^{10}$ reporting that $\mathrm{N}$-acetylcysteine reduced the incidence of CIN in patients undergoing computed tomography, generated considerable enthusiasm regarding this low cost and easy to use drug. That study randomised treatment of 83 patients who had serum creatinine $\geqslant 106.08 \mu \mathrm{mol} / \mathrm{l}$ (or $\mathrm{CrCl}<50 \mathrm{ml} / \mathrm{min}$ ) undergoing computed tomography with a small fixed intravenous contrast medium volume of $75 \mathrm{ml}$ and found an impressive $90 \%$ reduction in the relative risk of CIN. After that study, additional clinical trials carried out in patients undergoing coronary angiography, PCI, or both had, however, controversial results. ${ }^{11-19}$ Although in a meta-analysis $\mathrm{N}$-acetylcysteine has been shown to prevent CIN in patients with renal functional impairment undergoing cardiac catheterisation, the absence of small negative trials is a possible bias that may have influenced these findings as recognised by the authors. ${ }^{20}$ In addition, a recent systematic review by Kshirsagar and colleagues $^{22}$ suggested that the role of $N$-acetylcysteine in the prevention of CIN has yet to be defined.

The conflicting results between our study and previous studies may also be explained by the differences in the contrast media used. Our investigation was conducted with an ionic low osmolality contrast medium, which is used in almost half of PCIs performed outside the USA. ${ }^{23}$ All others studies used non-ionic contrast media. In experimental studies, ionic low osmolality contrast media and non-ionic contrast media have caused different degrees of renal hypoxia and structural damage in the tubule cells. ${ }^{24}{ }^{25}$ It is conceivable that $\mathrm{N}$-acetylcysteine has distinctive effects on preventing nephrotoxicity caused by various contrast agents.

In the present study the serum baseline creatinine concentration in the placebo group was slightly lower, with a borderline significance, than in the $N$-acetylcysteine group, although the estimated $\mathrm{CrCl}$ was similar in both groups. We assume that baseline creatinine concentrations did not influence the effect of $\mathrm{N}$-acetylcysteine as indicated by multivariate analysis.

The incidence of CIN in our investigation of patients with moderate renal failure (mean baseline creatinine $117.57 \mu \mathrm{mol} / \mathrm{l}$ ) was $10.3 \%$, similar to that found in other studies of patients at risk. ${ }^{11}{ }^{14}$ These patient characteristics do not allow us to draw definite conclusion about the effect of $N$-acetylcysteine on very high risk patients (creatinine $\geqslant 176.8 \mu \mathrm{mol} / \mathrm{l}$ ), even with the subgroup and multivariate analysis performed. However, considering that only $4 \%$ of the population undergoing cardiac catheterisation has serum creatinine concentrations above $176.8 \mu \mathrm{mol} / \mathrm{l},{ }^{2}$ the present findings can be applied to the majority of patients undergoing cardiac catheterisation at risk for developing CIN.

Table 4 Incidence of $\mathrm{CIN}$ in the predefined subgroups

\begin{tabular}{lllllll}
\hline & Number & N-acetylcysteine & Placebo & OR & 95\% Cl & p Value \\
\hline Diabetes mellitus & 81 & $10.0 \%$ & $9.8 \%$ & 1.02 & 0.27 to 4.42 & 1.00 \\
No diabetes mellitus & 75 & $10.8 \%$ & $10.5 \%$ & 1.03 & 0.23 to 4.46 & 1.00 \\
Creatinine $\geqslant 132.6 \mu \mathrm{mol} / \mathrm{l}$ & 46 & $15.4 \%$ & $20.0 \%$ & 0.72 & 0.15 to 3.35 & 0.71 \\
Creatinine $<132.6 \mu \mathrm{mol} / \mathrm{l}$ & 110 & $7.8 \%$ & $6.8 \%$ & 1.17 & 0.27 to 4.93 & 1.00 \\
Contrast $\leqslant 1.5 \mathrm{ml} / \mathrm{kg}$ & 96 & $11.4 \%$ & $9.6 \%$ & 1.20 & 0.32 to 4.46 & 1.00 \\
Contrast $>1.5 \mathrm{ml} / \mathrm{kg}$ & 60 & $9.4 \%$ & $11.5 \%$ & 0.79 & 0.14 to 4.30 & 1.00 \\
\hline
\end{tabular}

$\mathrm{Cl}$, confidence interval; OR, odds ratio. 
The dose and route of administration of $\mathrm{N}$-acetylcysteine in our study were the same as those in the majority of previous studies: $600 \mathrm{mg}$ orally every 12 hours for two days, beginning the day before the procedure. Recently, intravenous administration of $N$-acetylcysteine has been shown to be effective in an initial study of only 80 patients. ${ }^{26}$ However, a larger clinical trial with 425 patients did not find a benefit. ${ }^{27}$

\section{Secondary end points}

Once the rise of creatinine is a surrogate end point, we also investigated the effect of oral $\mathrm{N}$-acetylcysteine on hard clinical end points (in hospital death, length of hospitalisation, and need for haemodialysis). The study of Kay and colleagues ${ }^{14}$ was the only one that investigated the length of hospital stay as a secondary end point. Our study did not shown any benefit of $\mathrm{N}$-acetylcysteine on in-hospital death, need for dialysis, and length of hospital stay. The high inhospital mortality observed in this study may be explained by the high cardiovascular risk of the patients. More than half of the study patients had multivessel disease and some patients underwent surgical revascularisation during the study period.

\section{Subgroups}

$\mathrm{N}$-acetylcysteine was not effective in preventing CIN in any of the predefined subgroups. Diabetes mellitus is a well known independent risk factor for CIN. ${ }^{28}$ Post hoc analysis of a previous study indicated that $N$-acetylcysteine may effectively prevent CIN in patients with diabetes. ${ }^{14}$ In our study the predefined subgroup analysis of 81 diabetic patients indicated that $N$-acetylcysteine was not effective, independently of the presence or absence of diabetes mellitus. In another post hoc analysis, ${ }^{12} \mathrm{~N}$-acetylcysteine provided protection for patients receiving $<140 \mathrm{ml}$ of contrast medium during angiography. Our study did not confirm this finding.

\section{Clinical implications}

This is the first multicentre study evaluating the role of oral $\mathrm{N}$-acetylcysteine in preventing CIN in patients undergoing cardiac catheterisation with an ionic low osmolality contrast medium. Our major finding was that $N$-acetylcysteine did not prevent acute renal failure in patients at low to moderate risk of CIN.

On the basis of these findings, we believe that the use of $\mathrm{N}$ acetylcysteine to prevent CIN in this patient population should not be encouraged. The recommended measures for preventing CIN continue to be appropriate hydration and the use of a small volume of contrast in patients at low to moderate risk of CIN undergoing cardiac catheterisation with an ionic low osmolality contrast medium remain.

\section{Limitation of the study}

A potential limitation of this study was that serum creatinine was only measured 48 hours after the procedure. Although most clinical trials on preventive measures for CIN have assessed creatinine during that period and creatinine usually increases 24 hours after exposure and peaks within 48-72 hours, a later increase in serum creatinine may have passed unnoticed in some patients. The $\mathrm{CrCl}$ was estimated by the Cockcroft-Gault formula, which is widely used in clinical practice and in clinical trials; however, it is not a formal measurement of $\mathrm{CrCl}$.

The study sample size was calculated aiming at reaching statistical difference in primary outcomes. Therefore, although no trends were observed in the subgroups, our study has a limited statistical power for this analysis.

\section{Conclusion}

The use of $\mathrm{N}$-acetylcysteine was not effective in preventing $\mathrm{CIN}$ in patients at low to moderate risk undergoing cardiac catheterisation with an ionic low osmolality contrast medium.

\section{Authors' affiliations}

V O Gomes*, P Caramori*, R Lasevitch, L C Bodanese, A Araújo, P Nery, A Brizolara, Division of Interventional Cardiology, Hospital São Lucas, Pontifícia Universidade Católica-RS, Porto Alegre, Brazil

C E Poli de Figueredo, Division of Nephrology, Hospital São Lucas, Pontifícia Universidade Católica-RS, Porto Alegre, Brazil

A P Röedel, A P Caramori, Division of Cardiology, Hospital de Clínica, Porto Alegre, Brazil

F S Brito Jr**, Division of Interventional Cardiology, Hospital Israelita Albert Einstein, São Paulo, Brazil

H G Bezerra, Division of Interventional Cardiology, Hospital São Camilo, São Paulo, Brazil

*Also the Postgraduate Course of Medicine, Federal University of Rio Grande do Sul, Porto Alegre, Brazil

**Also the Division of Interventional Cardiology, Hospital São Camilo, São Paulo

\section{REFERENCES}

1 lakovou I, Danges G, Lansky AJ, et al. Incidence, predictors, and economic impact of contrast induced nephropathy: results in 8,628 patients treated with percutaneous coronary interventions [abstract]. J Am Coll Cardiol 2002;39(suppl A):2A.

2 Rihal CS, Textor SC, Grill DE, et al. Incidence and prognostic importance of acute renal failure after percutaneous coronary intervention. Circulation 2002; 105:2259-64.

3 Gomes VO, Blaya P, Poli de Figueiredo CE, et al. Contrast-media induced nephropathy in patients undergoing coronary angiography. J Invasive Cardiol 2003;15:304-10.

4 Solomon R, Werner C, Mann D, et al. Effects of saline, mannitol, and furosemide on acute decreases in renal function induced by radiocontrast agents. N Engl J Med 1994;331:1416-20.

5 Barrett BJ, Carlisle EJ. Metaanalysis of the relative nephrotoxicity of high- and low-osmolality iodinated contrast media. Radiology 1993;188:171-8.

6 Weisberg LS, Kurnik PB, Kurnik BR. Radiocontrast-induced nephropathy in humans: role of renal vasoconstriction. Kidney Int 1992;41:1408-15.

7 Safirstein R, Andrade L, Vieira JM. Acetylcysteine and nephrotoxic effects of radiographic contrast agents: a new use for an old drug. $N$ Engl J Med 2000;343:209-12.

8 Bakris GL, Lass N, Gaber AO, et al. Radiocontrast medium-induced declines in renal function: a role for oxygen-free radicals. Am J Physiol 1990;258:F115-20.

9 Baliga R, Ueda N, Walker PD, et al. Oxidant mechanisms in toxic acute renal failure. Am J Kidney Dis 1997;29:467-77.

10 Tepel N, Van der Giet N, Schwarzfeld C, et al. Prevention of radiographiccontrast-agent-induced reductions in renal function by acetylcysteine. N Engl J Med 2000;343:180-4

11 Diaz-Sandoval L, Kosowsky B, Losordo D. Acetylcysteine to prevent angiography-related renal tissue injury (APART trial). Am J Cardiol 2002;89:356-8.

12 Briguori $C$, Manganelli F, Scarpato $P$, et al. Acetylcysteine and contrast agentassociated nephrotoxicity. J Am Coll Cardiol 2002;40:298-303.

13 Durham JD, Caputo C, Dokko J, et al. A randomized controlled trial of Nacetylcysteine to prevent contrast nephropathy in cardiac angiography. Kidney Int 2002;62:2202-7.

14 Kay J, Chow WH, Chan TM, et al. Acetylcysteine for prevention of acute deterioration of renal function following elective coronary angiography and intervention: a randomized controlled trial. JAMA 2003;289:553-8.

15 Boccalandro F, Amhad M, Smalling R, et al. Oral acetylcysteine does not protect renal function from moderate to high doses of intravenous radiographic contrast. Catheter Cardiovasc Interv 2003;58:336-41.

16 Shyu KG, Cheng JJ, Kuan P. Acetylcysteine protects against acute renal damage in patients with abnormal renal function undergoing a coronary procedure. J Am Coll Cardiol 2002;40:1383-8

17 Allaqaband S, Tumuluri R, Malik AM. Prospective randomized study of Nacetylcysteine, fenoldopam, and saline for prevention of radiocontrast induced nephropathy. Catheter Cardiovasc Interv 2002;57:279-83.

18 Oldemeyer J, Biddle P, Wurdeman, et al. Acetylcysteine in the prevention of contrast-induced nephropathy after angiography. Am Heart J 2003;146:e23.

19 Fung J, Szeto C, Wilson W, et al. Effect of $\mathrm{N}$-acetylcysteine for prevention of contrast nephropathy in patients with moderate to severe renal insufficiency: a randomized trial. Am J Kidney Dis 2004;43:801-8.

20 Birck R, Kzrossok S, Markowetz F. Acetylcysteine for prevention of contrast nephropathy: meta-analysis. Lancet 2003;362:598-603.

21 Cockcroft DW, Gault MH. Prediction of creatinine clearance from serum creatinine. Nephron 1976;16:31-41.

22 Kshirsagar A, Poole C, Mottl A, et al. N-acetylcysteine for the prevention of radiocontrast induced nephropathy: a meta-analysis of prospective controlled trials. J Am Soc Nephrol 2004;15:761-9. 
23 Batchelor WB, Granger CB, Kleiman NS, et al. A comparison of ionic versus nonionic contrast medium during primary percutaneous transluminal coronary angioplasty for acute myocardial infarction (GUSTO Ilb). Am J Cardiol 2000;85:692-7.

24 Lancelot $\mathbf{E}$, Idee JM, Lacledere $C$, et al. Effects of two dimeric iodinated contrast media on renal medullary blood perfusion and oxygenation in dogs. Invest Radiol 2002;37:368-75.

25 Caglar Y, Mete VO, Kaya M. Ultrastructural evaluation of the effects of the contrast media on the rat kidney. J Submicrosc Cytol Pathol 2001;33:443-51.
26 Baker C, Wragg A, Kumar S, et al. A rapid protocol for the prevention of contrast-induced renal dysfunction: the RAPPID study. J Am Coll Cardiol 2003;41:2114-8.

27 Webb JG, Pate GE, Levin A, et al. Intravenous acetylcysteine in the prevention of contrast-induced nephropathy: a randomized trial. Can J Cardiol 2003; 19(suppl A):138A.

28 McCullough PA, Wolyn R, Rocher LL, et al. Acute renal failure after coronary intervention: incidence, risk factors and relationship to mortality. Am J Med 1997; 103:368-75.

\section{IMAGES IN CARDIOLOGY}

doi: $10.1136 /$ hrt.2004.050716

\section{Transoesophageal echocardiography for the identification of a giant aortic thrombus}

A 76 year old man presented with severe abdominal pain vomiting, and signs of lower right limb ischaemia. The patient had no previous medical history but smoked cigarettes. The ECG and transthoracic echocardiography showed no significant abnormality. Computerised tomography of his abdomen demonstrated multiple renal and splenic infarcts. The patient underwent emergency right femoral embolectomy and was anticoagulated with intravenous heparin.

A transoesophageal echocardiogram was requested. This revealed a normal heart with no evidence of intracardiac shunt. The descending aorta was visualised at the end of the procedure and there was a large pedunculated thrombus measuring $4 \times 2 \mathrm{~cm}$ (panel $\mathrm{A}$, video clip l) (to view video footage, visit the Heart website-http://www.heartjnl.com/ supplemental). Thrombolysis was considered, but in view of the risk of precipitating further emboli, the patient was anticoagulated with warfarin. The patient remained free from further embolic events. Transoesophageal echocardiography three months later revealed a smooth, posteriorly located plaque in the descending aorta (fig 2) with no evidence of residual thrombus.

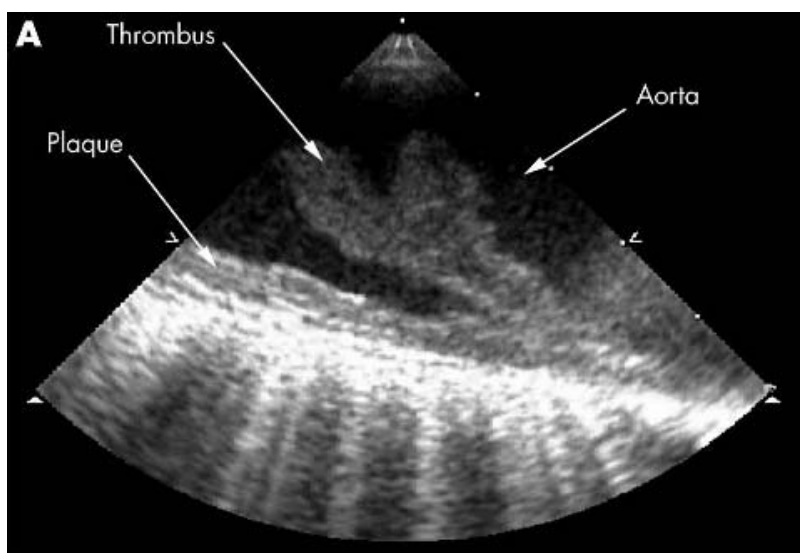

This report demonstrates the additional value of transoesophageal echocardiography in identifying a source of arterial embolisation. It is recognised that aortic atheromatous plaques are a potential source of embolism. Overlying aortic thrombi are a rare but potentially underestimated source of systemic emboli. Undiagnosed and untreated, they may have catastrophic consequences. In this case, although transthoracic echocardiography was normal, a giant thrombus in the descending aorta was found on a transoesophageal study. Transoesophageal echocardiography should be considered when there is high level of suspicion of a cardiac source of embolism.

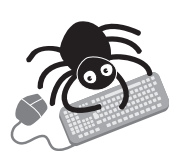

To view video clip visit the Heart website-http:// www.heartinl.com/supplemental

I H Mirza

A R J Mitchell

J Timperley

mitcharj@doctors.org.uk

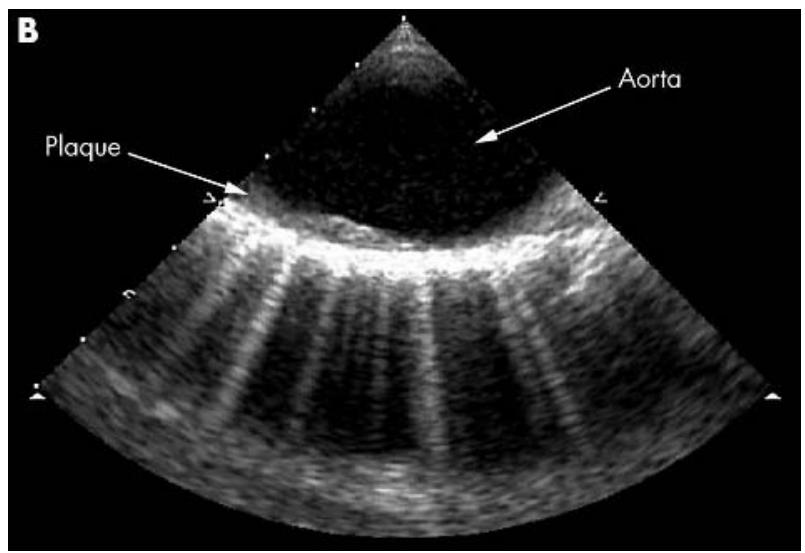

